\title{
Response of free-standing graphene monolayer exposed to ultrashort intense XUV pulse from free-electron laser
}

Cite as: J. Chem. Phys. 154, 204706 (2021); https://doi.org/10.1063/5.0041261

Submitted: 21 December 2020 . Accepted: 09 May 2021 . Published Online: 27 May 2021

\footnotetext{
(D) N. Medvedev, (D) H. Noei, S. Toleikis, and (D) B. Ziaja
}

\section{COLLECTIONS}

Paper published as part of the special topic on Special Collection in Honor of Women in Chemical Physics and Physical Chemistry

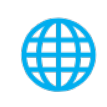

\section{Challenge us.}

What are your needs for periodic signal detection?

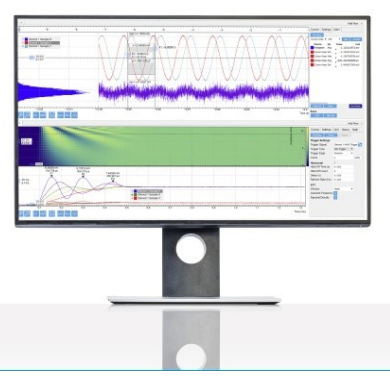

- Zurich

Instruments 


\title{
Response of free-standing graphene monolayer exposed to ultrashort intense XUV pulse from free-electron laser
}

\author{
Cite as: J. Chem. Phys. 154, 204706 (2021); doi: 10.1063/5.0041261 \\ Submitted: 21 December 2020 - Accepted: 9 May 2021 • \\ Published Online: 27 May 2021
}

N. Medvedev, ${ }^{1,2, a)}$ (D) H. Noei, ${ }^{3}$ (D) S. Toleikis, ${ }^{3}$ and B. Ziaja ${ }^{4,5, a)}$ (D)

\begin{abstract}
AFFILIATIONS
${ }^{1}$ Institute of Physics, Czech Academy of Sciences, Na Slovance 2, Prague 8 18221, Czech Republic

${ }^{2}$ Institute of Plasma Physics, Czech Academy of Sciences, Za Slovankou 3, 18200 Prague 8, Czech Republic

${ }^{3}$ Deutsches Elektronen-Synchrotron DESY, Notkestraße 85, 22607 Hamburg, Germany

${ }^{4}$ Center of Free-Electron Laser Science CFEL, Deutsches Elektronen-Synchrotron DESY, 22607 Hamburg, Germany

${ }^{5}$ Institute of Nuclear Physics, Polish Academy of Sciences, Radzikowskiego 152, 31-342 Krakow, Poland
\end{abstract}

Note: This paper is part of the JCP Special Collection in Honor of Women in Chemical Physics and Physical Chemistry.

a) Authors to whom correspondence should be addressed: nikita.medvedev@fzu.cz and ziaja@mail.desy.de

\begin{abstract}
The response of a free-standing graphene monolayer exposed to a few tens of femtoseconds long extreme ultraviolet (XUV) pulse was studied theoretically in order to analyze and compare contributions of various mechanisms to the graphene damage, understood here as a global atomic disintegration. Our simulation results indicate that nonthermal disintegration of the atomic structure is the predominant damage mechanism for a free-standing graphene layer. Only at high absorbed doses, charge-induced disintegration of the graphene structure prevails. We also demonstrate that the progressing damage can be probed by femtosecond optical pulses in the soft UV regime ( $4 \mathrm{eV}$ photon energy). The achieved quantitative understanding of the damage mechanisms may enable a better control of graphene-based devices when they are exposed to X-ray radiation, as well as an efficient processing of graphene layers with ultrashort intense XUV pulses.
\end{abstract}

Published under license by AIP Publishing. https://doi.org/10.1063/5.0041261

\section{INTRODUCTION}

Free-electron lasers (FELs) produce femtosecond light pulses with photons of extreme ultraviolet (XUV)/soft-x-ray up to hard $\mathrm{X}$-ray energies. ${ }^{1-3}$ Their intensities are sufficient to damage solid materials in a single shot ${ }^{4}$ or even to create warm dense matter ${ }^{5}$ or plasma. ${ }^{6}$ The unique property of $\mathrm{x}$-ray irradiation is that the geometry of the $\mathrm{x}$-ray affected volume in a material can be precisely shaped via an adjustment of $\mathrm{x}$-ray beam parameters such as photon energy, pulse fluence, beam focus, incidence angle, and polarization for a specific material. In addition, due to a very short FEL-matter interaction time, the heat diffusion to the surrounding material layer as well as the respective secondary material damage are significantly reduced when compared to optical pulse irradiation. This offers promising prospects for controlled processing of material surfaces. As the recent study showed, ${ }^{7}$ the laser fluences needed to create such surface modifications are relatively low.
The interaction of $x$-ray FEL pulses with solid bulk materials and on material surfaces has been extensively studied, both experimentally $y^{8,9}$ and theoretically. ${ }^{10-13}$ Experimental studies of damage induced by intense $\mathrm{x}$-ray pulses lead to the development of accurate $x$-ray beam diagnostics. ${ }^{14,15}$ Continuously improving theoretical modeling enables us to identify various mechanisms of structural transformations within irradiated solids induced by $\mathrm{x}$ rays. ${ }^{10,16}$

However, the response of free-standing two-dimensional (2D) materials (such as graphene) to ultrashort $\mathrm{x}$-ray irradiation has not been investigated systematically, so far. A 2D material represents a limiting case of a material layer, which is atomically thin. Graphene is an atomically thin sheet of $\mathrm{sp}^{2}$-bonded carbon atoms organized into a honeycomb structure. ${ }^{17}$ Over the last few years, it has been attracting much attention due to its unique properties, such as high electron mobility and thermal conductivity. It is also a promising candidate for a new generation of electronic devices. 
Graphene usually exhibits high resistance against ionizing radiation. ${ }^{19,20}$ However, this resistance depends on the sample holding conditions and on the irradiation type. For instance, the stabilization of a graphene layer in the third dimension (e.g., via its interaction with an underlying substrate or via mutual interactions between graphene sheets within a few-layer sample) increases robustness under soft x-ray irradiation. ${ }^{21}$ However, in some cases, an interaction with a substrate may result in damage to graphene. This occurs if, e.g., the expansion of the irradiated substrate creates a sufficiently high pressure to detach graphene from the substrate and even to disrupt it. Such effects were, indeed, reported after irradiation of graphene-substrate samples with swift heavy ions $^{22}$ and with XUV/soft-x-ray pulses. ${ }^{23}$ In a lower-intensity irradiation regime, multiple exposure to XUV or electron beams is required until point defects accumulate and induce macroscopic phase transitions toward amorphization. ${ }^{24}$ Intense optical laser illumination results in an ablation of graphene on a thin glass substrate, whereas optical pulse fluences below the damage threshold induce breaking of graphene bonds and formation of defects. ${ }^{25}$

However, it seems difficult to macroscopically damage a freestanding graphene monolayer with $\mathrm{x}$-ray irradiation. ${ }^{26}$ It was confirmed in a recent $\mathrm{x}$-ray FEL experiment, where a free-standing graphene layer was used as a support for biosamples during imaging studies, performed with photons of $8 \mathrm{keV}$ energy. ${ }^{26}$ The main reason can be the low absorption of radiation energy by a monolayer. When $\mathrm{x}$ rays irradiate a bulk material, photoabsorption occurs not only on the material surface but also deeper in the material. The photoabsorbed energy is then carried by the released photo- and Auger electrons and distributed further within the solid through electronic collisional processes. ${ }^{10}$ This induces a volumetric distribution of the absorbed energy in the solid, with the effective radiation penetration depth dependent on the $\mathrm{x}$-ray grazing angle. ${ }^{13}$

If only the region close to the surface should be intensely irradiated in a bulk material, (i) the $\mathrm{x}$-ray grazing angle should be small and (ii) the $\mathrm{x}$-ray radiation should be s-polarized. ${ }^{27}$ The reason for the first condition is that the penetration depth in the material, i.e., the depth at which on average $~ 63 \%$ of the photons that entered into the material become absorbed, ${ }^{13}$ decreases with the decreasing grazing angle. Still, reasonably low material reflectivity at such grazing angles enables to transfer much of the incoming photon flux into the material. This changes if the grazing angle decreases below a critical angle specific for the material and photon energy/wavelength. The reflectivity then approaches $100 \%$, and the radiation energy absorption in the material becomes inefficient.

Concerning the second condition, as photoelectrons are primarily emitted along the direction corresponding to the polarization vector of the $\mathrm{x}$-ray radiation, the distribution of secondary electrons triggered by an impact of the photoelectron also "peaks" around this direction. Therefore, choosing the s-polarization of $\mathrm{x}$ rays triggers a buildup of the electron distribution along the direction parallel to the material surface, preventing these electrons from penetrating deeply into the material. This, in addition, restricts the radiation-affected volume in the material. ${ }^{2}$

These mechanisms of energy deposition do not work efficiently for an irradiated monolayer, as in this case, the x-ray excited energetic electrons quickly leave the sample and, therefore, do not distribute their energy further, through collisional processes, in the sample. In particular, photoelectrons (which are then emitted with the highest probability in-plane with the layer) scatter only a few times before leaving the layer. X-ray energy absorption can then be only increased by an increase in the incoming photon flux. It should then arrive under normal incidence to the material surface, in order to provide the highest intensity on the graphene layer. ${ }^{29,30}$

An insight into the mechanisms of damage in this specific case requires a dedicated study. Therefore, here we investigate in detail the effect of ultrafast XUV FEL irradiation on a free-standing graphene sheet, using computer simulations. We demonstrate that, unlike other types of irradiation, femtosecond intense $\mathrm{x}$ rays trigger specific macroscopic damage mechanisms-namely, nonthermal damage and/or Coulomb explosion. We expect that the achieved precise understanding of the damage mechanisms in $\mathrm{x}$-ray irradiated graphene can lead to a better control of graphene-based devices in applications, in which they are exposed to x-ray radiation. A control over X-ray-induced damage processes in graphene can also enable an efficient processing of graphene samples with ultrashort intense $\mathrm{X}$-ray pulses.

\section{METHODS}

For the simulations of $\mathrm{x}$-ray-induced damage processes in a free-standing graphene sheet, we applied our hybrid code XTANT (X-ray-induced Thermal And Nonthermal Transitions ${ }^{10}$ ). The code has been developed specifically for studying the response of solid materials to ultrafast $\mathrm{x}$-ray irradiation. It includes the following interconnected modules:

(a) Monte Carlo (MC) model, which traces the following processes: $\mathrm{x}$-ray photoabsorption; creation of energetic free electrons and of core holes (if the incoming $\mathrm{x}$-ray photon energy is sufficient to ionize core atomic levels); electron impact ionization and quasi-elastic electron scattering on atoms; emission of electrons in case of an open-surface modeling; and Auger decays of core holes. Photoabsorption cross sections, as well as any required rates and parameters, are extracted from the EPICS-2017 database. ${ }^{31}$ For modeling the electron impact ionization cross sections, the Binary-EncounterBethe (BEB) model is used. ${ }^{3}$

Each photo-, Auger-, or collisionally emitted electron is traced until its kinetic energy falls below a cutoff energy (here set to $10 \mathrm{eV}$ ). ${ }^{33}$ Electrons with energies below the cutoff energy form source terms for the kinetic equations describing populations of (slow) electrons in the valence and conduction bands. To model electron emission from the graphene sheet, we use the approximation of electron loss after a single collision. ${ }^{34}$ It implies that we remove an electron from the MC module after a single collision, if its kinetic energy after the scattering lies above the cutoff energy; if the energy falls below the cutoff energy, the electron joins the lowenergy electron fraction [see (b) below]. This approximation assumes that an emitted electron does not return to graphene, which is a reliable estimation for sufficiently fast electrons and low charges accumulated in the sample. Removal of electrons from the Monte Carlo module leaves a positive charge in the atomic system, which is accounted for by assigning average fractional charges ${ }^{34}$ to all atoms. 
(b) Rate equations are used to trace the distribution of lowenergy electrons. The equations describe electron populations on energy levels (molecular orbitals) within the valence and conduction bands. The actual orbital energies are calculated with the transferable tight binding (TB) parameterization [see (e) below]. Electron-electron scattering is assumed to instantaneously thermalize the low-energy part of the electronic system so that the distribution function always adheres to a Fermi-Dirac shape ${ }^{18}$ Energy exchange of the electronic system with the atomic system, leading to an increase in the atomic temperature, is traced with Boltzmann collision integrals (BCI).

(c) Boltzmann collision integrals (BCI) describe nonadiabatic interactions between the electrons and atoms using the "dynamical coupling" approach developed in. It calculates the probability of an electron transition between the energy levels, triggered by atomic displacements. Note that this approach does not rely on the accuracy of the phononic approximation for the atomic motion (periodic lattice, harmonic vibrations, etc.). Instead, it is applicable to any kind of atomic motion and configuration. This makes it also suitable for the strongly non-equilibrium conditions studied here.

(d) Atomic motion is traced within a molecular dynamics (MD) framework. Atomic potential energy surface is calculated within the transferable tight binding formalism. Additionally, the energy provided via quasi-elastic scattering of highenergy electrons on atoms (treated within the MC module) and via the nonadiabatic coupling of atoms to low-energy electrons (from BCI) is introduced into the atomic system at each time step via velocity scaling. Coulomb potential between the atoms, induced by the charges left after the electron emission, is added to the atomic potentials via a pairwise softly cut Coulomb term. ${ }^{34}$

(e) Transferable tight binding (TB) method is used to calculate the atomic potential energy surface forming covalent bonds in the solid. It also provides the corresponding forces, electronic band structure, and wave functions necessary for the evaluation of matrix elements ${ }^{35}$ entering BCI [see (c)] and the calculation of the complex dielectric function within the random phase approximation. ${ }^{36}$ For carbon, the transferable TB parameterization from Ref. 37 is used. The TB parameters do not depend on the electronic temperature, which is, of course, an approximation. However, TB applicability to electronically excited systems was confirmed for various systems such as diamond, graphite, amorphous carbon, and $\mathrm{C}_{60}$ under various irradiation conditions (for details, see the review ${ }^{10}$ and references therein). Changes in the atomic potential energy upon electronic excitation are "naturally" included within the TB module, thereby allowing us to model nonthermal effects, such as nonthermal melting and phase transitions, including ablation. ${ }^{10}$

All the modules are executed in parallel, so all the processes described above influence each other. The details on each individual module of the code, their interconnections, and the details of the numerical algorithm can be found in Ref. 10. To model optical properties of the target, we employ the random phase approximation, in which the electronic populations, as well as the electron energy levels and oscillator strengths, are updated at each time step of the simulation in XTANT. ${ }^{36}$

The simulation box contained 200 atoms with periodic boundary conditions in the $(\mathrm{X}, \mathrm{Y})$ plane (in which the graphene sheet was aligned) and free boundaries along the $\mathrm{Z}$ axis. For modeling the FEL pulse irradiation, we used temporal Gaussian pulses of $30 \mathrm{fs}$ FWHM pulse duration depositing various radiation doses on the graphene layer. The XUV irradiation regime was considered, as the photoabsorption cross section for carbon atoms is the largest in this $\mathrm{x}$-ray photon energy regime. ${ }^{38}$

\section{RESULTS}

As an example, we show the evolution of a graphene monolayer after its irradiation with a high intensity XUV FEL pulse (with a photon energy of $30 \mathrm{eV}$ ), yielding an average absorbed dose of $5 \mathrm{eV} /$ atom. Figure 1 presents atomic snapshots of damage progressing in free-standing graphene during a few hundreds of femtoseconds, simulated within a nonadiabatic scheme. Time $0 \mathrm{fs}$ corresponds here to the time instant when the $\mathrm{x}$-ray pulse had a maximal intensity. By the time of $300-500 \mathrm{fs}$, the sample disintegrates into fragments, mainly consisting of linear chains of carbon atoms. The damage can be observed both in the graphene plane and along the direction normal to the graphene plane. The latter indicates that the damage destroyed the initial 2D order in the material and evolved it into a three-dimensional (3D) structure. This evolution progresses with carbon atoms forming sp-bond arrangements (linear chains), reducing the number of atoms in the $\mathrm{sp}^{2}$-bonded configuration.

The results of our calculations indicate that two processes can induce the graphene damage, their specific contribution dependent on the x-ray dose absorbed by graphene. As damage, we understand here a global disintegration of the atomic structure within the graphene sheet and not a creation of local defects. The respective contributing processes are as follows:

(i) nonthermal disintegration of the atomic structure resulting from the modification of the interatomic potentials from an attractive to a repulsive one. This modification is due to the electronic excitation following an x-ray impact, which brings the electrons from the bonding orbitals of the valence band to the antibonding states in the conduction band. If the excitation is strong enough, it can break chemical bonds between the atoms. ${ }^{39}$ In the case of irradiation with $30 \mathrm{eV}$ photons, the threshold for this bond breaking corresponds to an excitation of $\sim 4.5 \%$ of electrons from the valence band, occurring at $\sim 4.1 \mathrm{eV} /$ atom deposited dose. Below this dose, $\mathrm{x}$-ray irradiation induces only significant atomic vibrations and bending in graphene, but no ripping or hole formation are visible on the hundreds of femtoseconds time scales.

(ii) charge-induced disintegration of the atomic structure in the graphene monolayer. This mechanism starts to prevail at high absorbed doses when carbon atoms are quickly stripped of valence electrons, which then escape. As a result, the sample charges fast. The increasing Coulomb repulsion between the charged ions breaks the bonds and moves the ions apart. One can roughly estimate that the charge-induced 


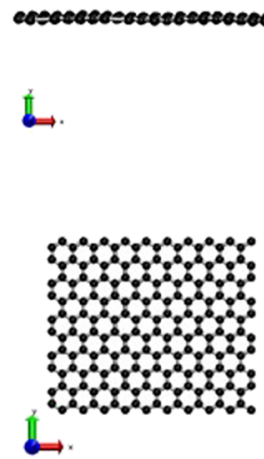

$0 \mathrm{fs}$

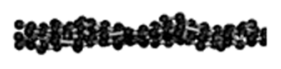

in.

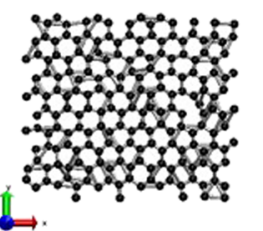

$100 \mathrm{fs}$
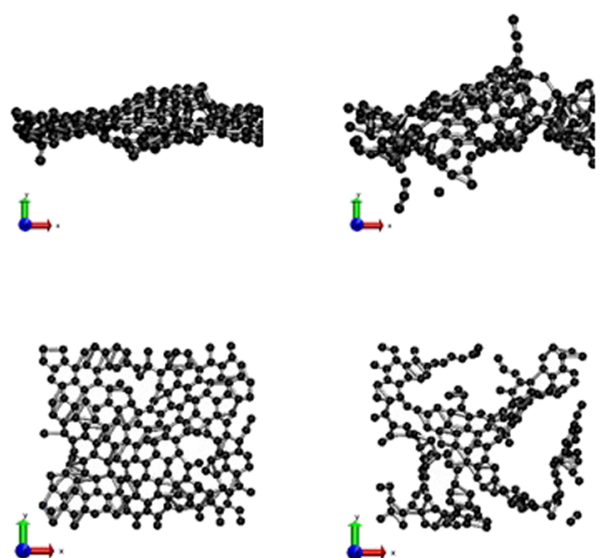

$200 \mathrm{fs}$

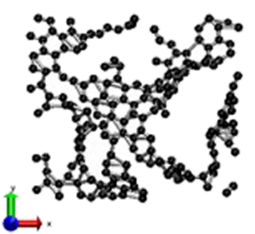

$300 \mathrm{fs}$

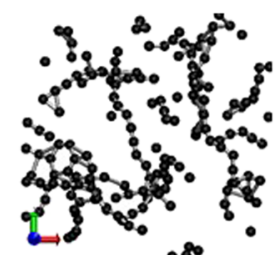

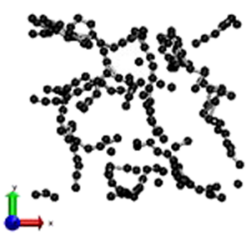

$500 \mathrm{fs}$

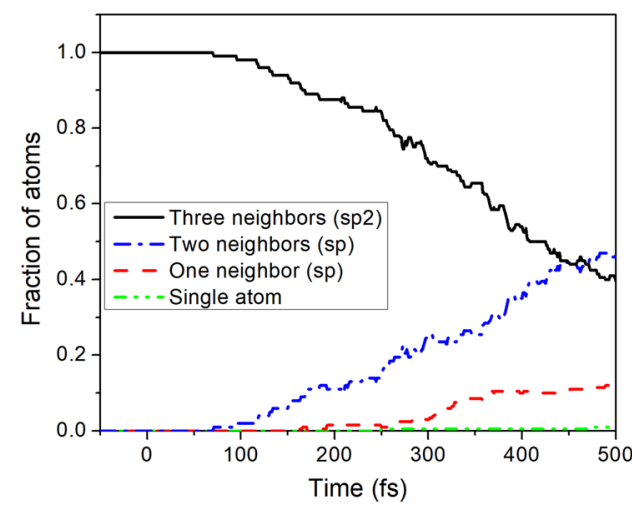

FIG. 1. (Top panel) XTANT ${ }^{10}$ simulations of $X U V$ irradiated graphene, with periodic boundaries along $X$ and $Y$ axes, and free boundary along the $Z$ axis. The supercell contained 200 carbon atoms. It was irradiated with an XUV FEL pulse ( $30 \mathrm{eV}$ photon energy, Gaussian temporal shape, $30 \mathrm{fs}$ FWHM) of high intensity, yielding, on average, $5 \mathrm{eV}$ deposited dose per atom. Time was counted from the maximum of the Gaussian FEL pulse. The top row of figures is the side view (along the $Z$ axis). The bottom row shows the projection on the $(X, Y)$ plane. (Bottom panel) Fraction of atoms with a given number of nearest neighbors, having $s p^{2}$ and $s p$ bonding, and monomers.

disintegration starts to dominate, when the average ionization degree per atom approaches $\sim+1$. This corresponds to the case in which every atom in the monolayer has been singly ionized and allows us to assume, similarly to Ref. 40, that the majority of $\mathrm{C}-\mathrm{C}$ bonds have been broken because both constituent carbon atoms were ionized.

In our study case presented in Fig. 1, the total number of excited electrons that left the graphene monolayer (estimated with our simulation tool) was $0.13-0.14$ per atom. This charge-induced Coulomb repulsion between the atoms was insufficient to break interatomic bonding and induce Coulomb explosion. This clearly indicates that the ion repulsion did not dominate the sample damage, and the electron-excitation induced damage (i.e., nonthermal damage) was the predominant mechanism of bond breaking. The approximation of fractional charges misses a possible effect of local bond breaking when two integer charges are located at the neighboring atoms. However, in our case, maximally one in ten atoms can have an integer charge. Therefore, the probability for the occurrence of a local bond breaking is low and does not affect our predictions on global damage. The approximation holds reasonably well for the fluences considered here, as long as the graphene layer is intact but may fail to describe the fragmented species at fluences significantly above the damage threshold. In any case, local damage effects are beyond the scope of the present paper, where we focus on the global damage process.

Another process that could potentially affect the damage is the progressing energy exchange between the electronic system and the atomic lattice. The calculated electron-ion coupling parameter in graphene is shown in Fig. 2. In the top panel of Fig. 2, it is presented for a two-dimensional system, assuming normalization of the energy exchange rate per two-dimensional area of the graphene sample. The XTANT results are compared with other available estimations from Refs. 41-43. The results of Ref. 42 were obtained for distorted graphene, which is probably one of the reasons for a significantly higher value of the predicted coupling parameter. They are 


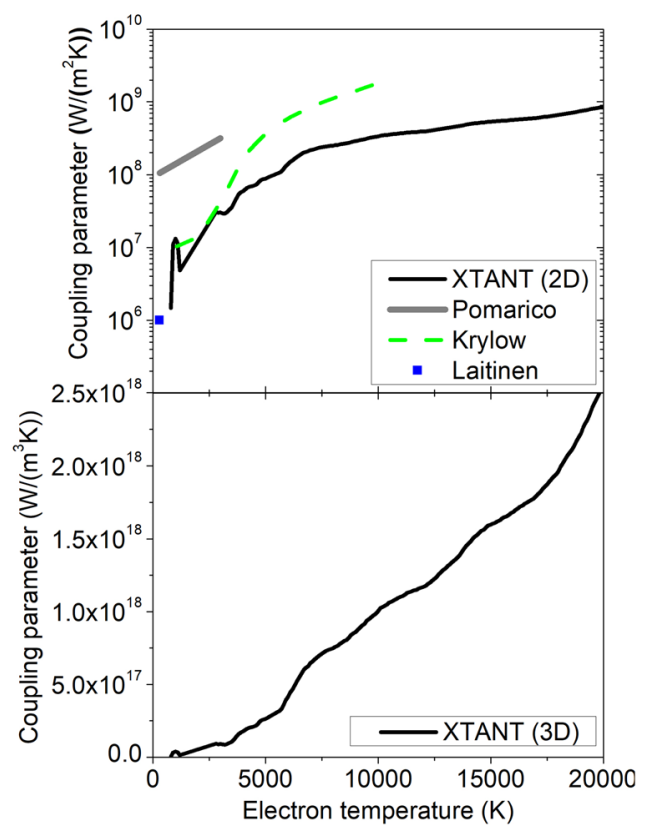

FIG. 2. Electron-ion (electron-phonon) coupling parameter calculated by $\mathrm{XTANT}^{10}$ in a single layer of free-standing graphene monolayer. Top panel: twodimensional coupling parameter, compared with the estimates by Laitinen et al.," by Pomarico et al., ${ }^{42}$ and by Krylow et al. ${ }^{43}$ Bottom panel: three-dimensional coupling normalized per thickness of the single layer assumed to be $3.35 \AA$.

also higher than those predicted by other simulations from Refs. 41 and 43.

We note that the electron-phonon coupling parameter calculated by Krylow et al..$^{43}$ raises with the increase in the electron temperature significantly faster than that predicted by XTANT. The reason for this is that Krylow et al. ${ }^{43}$ used Eliashberg formalism for the coupling calculations. It was demonstrated in Ref. 44 that Eliashberg formalism overestimates the coupling parameter in metals at high electronic temperatures in comparison with experimental data, whereas XTANT predicts values much closer to experiments. The problem of the overestimated coupling was noted already by Lin et al., ${ }^{45}$ which led to the need to rescale the coupling in several metals (for example, in $\mathrm{Al}$ ) in Ref. 45 . Note that the work, ${ }^{46}$ which claimed that the electron-phonon coupling in graphene is high, employed the two-temperature model to interpret their experimental data. The two-temperature model does not account for nonthermal (and Coulomb explosion) effects and, thus, cannot be used to reliably extract the electron-phonon coupling in situations where the named effects are significant. As we demonstrate below, these effects are, indeed, predominant in graphene. The electron-phonon coupling in graphene at high electronic temperatures ultimately requires validation by dedicated experiments.

In the bottom panel of Fig. 2, we also show a three-dimensional estimation, which is obtained by dividing the two-dimensional one by the thickness of the atomic layer, assumed to be $3.35 \AA$. This volume-normalized estimation is convenient for a comparison with bulk materials, e.g., from Refs. 44 and 45. One can see that the coupling parameter assumes a low value at near-room electronic temperatures. However, at higher temperatures, it increases almost linearly to the values much higher than those observed in most metals. $^{44}$

In order to evaluate the effect of electron-ion coupling on the damage in the graphene sheet in our study case (Fig. 1), we compared the results of the nonadiabatic calculation including the electron-ion coupling (reported above) with the results of the calculation performed within the Born-Oppenheimer (BO) approximation. The latter scheme excludes electron-ion coupling. The damage threshold obtained with the BO scheme is only slightly higher: $\sim 4.2 \mathrm{eV} /$ atom than that obtained with the nonadiabatic calculation: $\sim 4.1 \mathrm{eV} /$ atom. Such an insignificant increase in the damage threshold points toward an interpretation that heating atoms does not affect the predominant nonthermal mechanism of the damage at subpicosecond time scales. To become more specific, below we also analyze the predicted changes in electronic and atomic temperatures depicted in Fig. 3.

The disintegration of the atomic lattice due to atomic heating only (i.e., thermal melting) takes place at atomic temperatures of $\sim 4000 \mathrm{~K} .{ }^{47}$ Under the current irradiation conditions, this temperature is reached in the simulated nonadiabatic case at $\sim 150 \mathrm{fs}$ since time-zero $(0 \mathrm{fs})$. Similar time scales of electronic relaxation in irradiated graphene were reported in the experimental study. ${ }^{46}$ This supports the conclusion that the initial graphene damage is of mostly nonthermal nature and that the initial atomic temperature increase is a result of the progressing nonthermal damage, and not the cause of it. A similar effect was observed in the simulations of graphitization in diamond. ${ }^{10,11}$

We note that the lattice temperature in the case of $5 \mathrm{eV} /$ atom dose increases above the electronic one at times $\sim 300 \mathrm{fs}$. This is a result of ongoing increase in the atomic kinetic energy due to Coulomb repulsion. At such doses above the damage threshold, atoms gain energy faster than that the electronic temperature can "keep up." In contrast, already at a lower dose of $4.5 \mathrm{eV} /$ atom, the Coulomb repulsion is not strong enough, and the growth of the lattice temperature is predominantly due to the energy exchange between the electrons and the lattice. Therefore, both temperatures stay close after the equilibration (see Fig. 4).

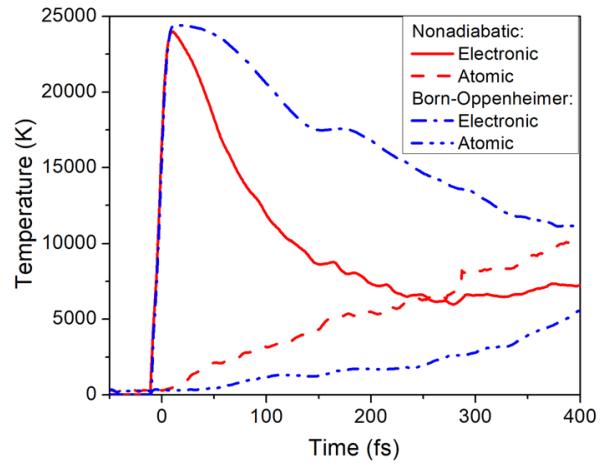

FIG. 3. XTANT predicted changes in electronic and atomic temperatures for the irradiated graphene monolayer. Irradiation parameters are the same as those in Fig. 1. 


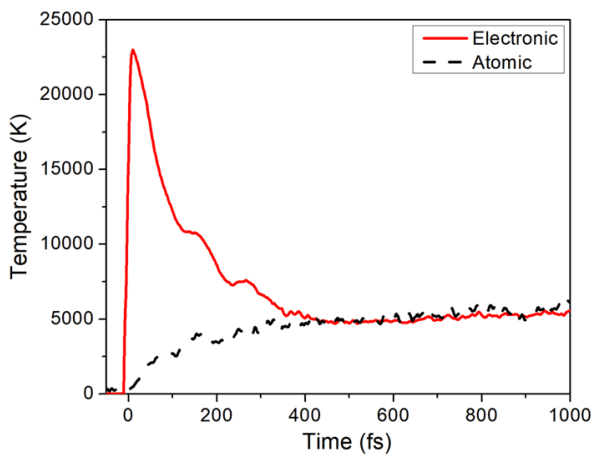

FIG. 4. Example of electron and ion temperature equilibration at the dose of $\sim 4.5 \mathrm{eV} / a$ tom, when the Coulomb repulsion between ions is still weak, and the growth of the lattice temperature is predominant due to the energy exchange between electrons and the lattice.

Finally, let us estimate the threshold fluence, F, for the nonthermal damage. Through the threshold fluence, we mean here the XUV pulse fluence arriving on the graphene monolayer. Knowing the damage threshold dose, $\mathrm{D}$, one can evaluate the corresponding damage threshold fluence for incoming photons under normal incidence from the following relation: $\mathrm{F}=\mathrm{D} / \sigma$, where $\sigma$ is the photoabsorption cross section for carbon atoms (here taken from Henke's tables ${ }^{48}$ ). We neglect here the effect of the reflected radiation, as the reflectivity of a carbon surface for XUV photons of $30 \mathrm{eV}$ energy is only $\sim 5 \%$. The result is plotted in Fig. 5. Due to the strong decrease in the photoabsorption cross section with the increasing $\mathrm{x}$-ray photon energy, one obtains the lowest values of the threshold fluence in the XUV regime. There is also a characteristic drop in the threshold fluence at the K-edge of carbon, which reflects the increase in the photoabsorption cross section at photon energies higher than the ionization potential of the $K$ shell, thereby reducing the damage threshold in terms of incoming fluence.

Note that the threshold fluence estimation intrinsically accounts for the energy loss due to the escape of energetic

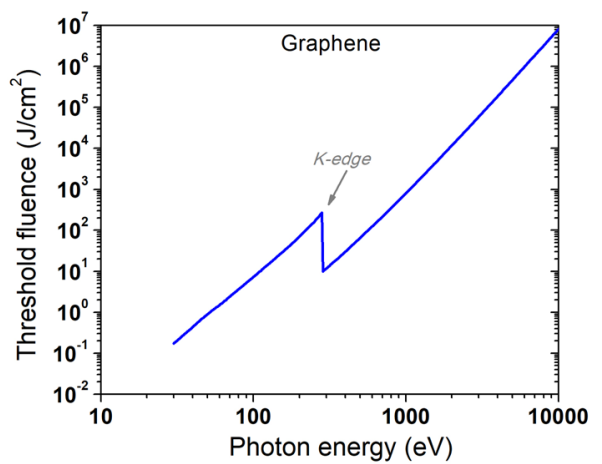

FIG. 5. Threshold fluence calculated for the graphene monolayer at various photon energies, assuming the damage threshold dose of $\sim 4 \mathrm{eV} /$ atom. electrons from the sample and the following sample charging. Therefore, this dose is much higher than the nonthermal damage threshold dose in bulk solids (usually $\leq 1 \mathrm{eV} /$ atom), where the energetic electrons do not escape from the sample, which then remain net-neutral. ${ }^{3}$

\section{DISCUSSION ON DIAGNOSTICS OF STRUCTURAL CHANGES}

Here, we will briefly discuss possible experimental diagnostics of the structural changes observed in the graphene. Concerning the initial and postmortem diagnostics of the graphene layer, Raman spectroscopy and x-ray diffraction are well-established tools. ${ }^{49-51}$ Unfortunately, Raman spectroscopy cannot be used as a time-resolved diagnostics tool able to follow ultrafast transient changes in the graphene structure. Time-resolved x-ray diffraction cannot be applied for graphene pumped by XUV FEL pulses. To explain the latter limitation, if the pump pulse would be in the XUV photon energy regime, and the usual split-and-delay $\mathrm{X}$-ray pump-X-ray probe scheme were applied, the XUV photons from the probe pulse could not generate a diffraction pattern of the resolution sufficient to follow bond deformation and breaking of graphene (cf. the interatomic distance in graphene of
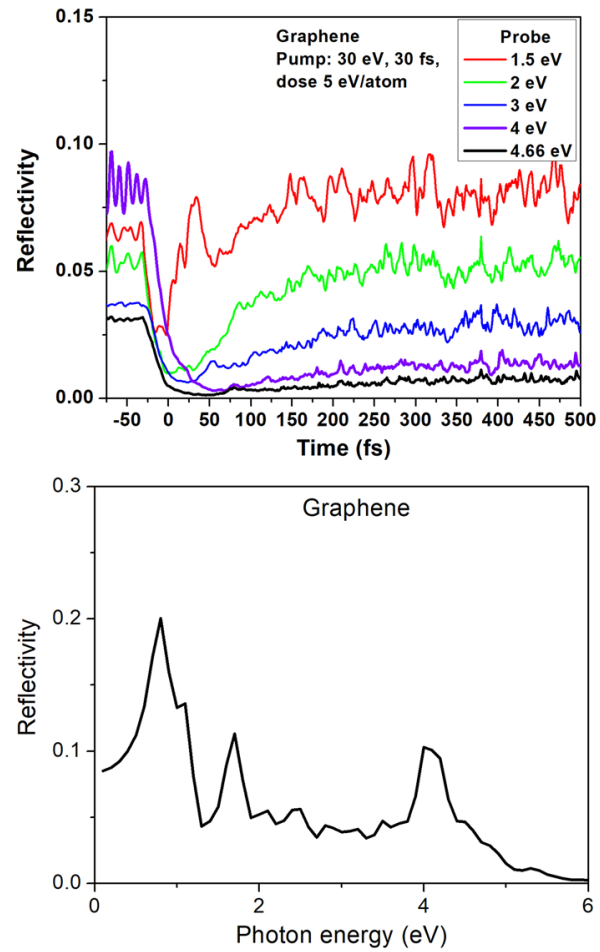

FIG. 6. (Top panel) Reflectivity for probe pulses of various photon energies, arriving under $45^{\circ}$ incidence angle to the graphene layer, calculated as a function of pump-probe delay time. (Bottom panel) Reflectivity spectrum calculated as a function of probing photon energy for probe pulses arriving under $45^{\circ}$ incidence angle to the undamaged graphene layer. Both calculations were performed with XTANT for $3 \times 3 \times 3$ k-points and 200 atoms in the simulation box. 
$\sim 0.142 \mathrm{~nm}$ ). A promising although technically challenging alternative could be an XUV pump-electron diffraction probe experiment (cf. Ref. 52), if sub-ps delay times for electron diffraction could be achieved.

However, an XUV pump-optical probe measurement can be considered as a time-resolved diagnostics tool for XUV irradiated graphene. In such an experiment, transient optical properties are monitored, which then change in response to XUV irradiation. ${ }^{12,29,30}$ Considering this scheme, we calculated with XTANT the transient reflectivity for an optical probe pulse, ${ }^{36}$ using our standard random-phase-approximation based approach that proved successful in Ref. 12. We assumed that after a certain delay since the impact of the XUV pulse, an (instantaneous) optical probe pulse arrived at $45^{\circ}$ incidence to the graphene monolayer. The probe pulse was assumed to have a low fluence in order to avoid any additional damaging effect on the sample. Various probe-pulse wavelengths were analyzed, examples of which are shown in Fig. 6.

Graphene is mostly transmissive in the optical wavelength range, with a little contribution of reflection. ${ }^{53,54}$ Nonetheless, Fig. 6 demonstrates that the optical reflectivity is mostly affected by the electronic excitations and not by the atomic motion during breakage of the graphene sheet. The strongest change in reflectivity was observed for the photon energy of $4 \mathrm{eV}$ (Fig. 6, top panel), whereas it was less sensitive at photon energies of $1.5-3 \mathrm{eV}$. This is explained by the presence of a small spectral peak at $\sim 4 \mathrm{eV}$ photon energy (see Fig. 6, bottom panel), which may shift or reduce during the electronic excitation. The measurement of optical reflectivity at an optical photon energy close to $4 \mathrm{eV}$ then seems a promising tool for the time-resolved diagnostics of irradiated graphene. Please note that we assumed here a temporally instantaneous optical pulse (delta-function-like). For specific experimental conditions, it has to be convolved with the optical pulse duration.

\section{CONCLUSIONS}

The response of a free-standing graphene monolayer exposed to a few tens of femtoseconds long XUV pulse was studied theoretically with the hybrid simulation tool XTANT. The tool treats all predominant processes in the electronic and atomic systems of graphene, following its intense XUV irradiation. This enabled us to analyze and compare the contribution of various mechanisms to the graphene damage, understood here as its global atomic disintegration.

Our results indicate that nonthermal disintegration of the atomic structure in graphene resulting from the modification of the interatomic potential from an attractive to a repulsive one is the predominant mechanism of the atomic disintegration in a free-standing graphene sheet. Only at high absorbed doses, charge-induced disintegration of graphene atomic structure prevails. This occurs when carbon atoms are quickly stripped of valence electrons, which then escape from the sample. As a result, the sample charges fast, and the increasing Coulomb repulsion between created ions breaks the interatomic bonds and moves the ions apart. The third process, the energy exchange between the electron system and the atomic lattice due to electron-ion coupling, does not contribute significantly to the damage progress at sub-ps times.
We demonstrated that the damage progress can be probed by femtosecond optical pulses. The optical reflectivity in the soft $\mathrm{UV}$ regime (at $\sim 4 \mathrm{eV}$ photon energy) is the most sensitive to electron excitation. The other promising diagnostics method, combining XUV pump irradiation and electron diffraction probe, could be applied if sub-ps resolution for electron diffraction could be reached.

Precise understanding of the damage mechanisms achieved with this study may lead to a better control of graphene-based devices when they are exposed to $\mathrm{x}$-ray radiation and enable an efficient processing of graphene samples with ultrashort intense $\mathrm{x}$-ray pulses.

\section{ACKNOWLEDGMENTS}

Partial financial support from the Czech Ministry of Education, Youth and Sports, Czech Republic (Grant Nos. LTT17015 and LM2015083) is gratefully acknowledged by N.M. This article/publication is based on the work within COST Action Grant No. CA 17126 "Towards understanding and modelling intense electronic excitation," supported by the COST (European Cooperation in Science and Technology).

The authors declare no competing interest.

\section{DATA AVAILABILITY}

The data that support the findings of this study are available from the corresponding author upon reasonable request.

\section{REFERENCES}

${ }^{1}$ J. Rossbach, J. R. Schneider, and W. Wurth, Phys. Rep. 808, 1 (2019).

${ }^{2}$ C. Bostedt, S. Boutet, D. M. Fritz, Z. Huang, H. J. Lee, H. T. Lemke, A. Robert, W. F. Schlotter, J. J. Turner, and G. J. Williams, Rev. Mod. Phys. 88, 015007 (2016).

${ }^{3}$ D. Pile, Nat. Photonics 5, 456 (2011).

${ }^{4}$ J. B. Pelka, R. Sobierajski, D. Klinger, W. Paszkowicz, J. Krzywinski, M. Jurek, D. Zymierska, A. Wawro, A. Petroutchik, L. Juha, V. Hajkova, J. Cihelka, J. Chalupsky, T. Burian, L. Vysin, S. Toleikis, K. Sokolowski-Tinten, N. Stojanovic, U. Zastrau, R. London, S. Hau-Riege, C. Riekel, R. Davies, M. Burghammer, E. Dynowska, W. Szuszkiewicz, W. Caliebe, and R. Nietubyc, Radiat. Phys. Chem. 78, S46 (2009).

${ }^{5}$ F. Graziani, M. P. Desjarlais, R. Redmer, and S. B. Trickey, Frontiers and Challenges in Warm Dense Matter (Springer-Verlag New York, Inc., New York, 2014).

${ }^{6}$ S. M. Vinko, O. Ciricosta, B. I. Cho, K. Engelhorn, H.-K. Chung, C. R. D. Brown, T. Burian, J. Chalupský, R. W. Falcone, C. Graves, V. Hájková, A. Higginbotham, L. Juha, J. Krzywinski, H. J. Lee, M. Messerschmidt, C. D. Murphy, Y. Ping, A. Scherz, W. Schlotter, S. Toleikis, J. J. Turner, L. Vysin, T. Wang, B. Wu, U. Zastrau, D. Zhu, R. W. Lee, P. A. Heimann, B. Nagler, and J. S. Wark, Nature 482, 59 (2012).

${ }^{7}$ T.-H. Dinh, N. Medvedev, M. Ishino, T. Kitamura, N. Hasegawa, T. Otobe, T. Higashiguchi, K. Sakaue, M. Washio, T. Hatano, A. Kon, Y. Kubota, Y. Inubushi, S. Owada, T. Shibuya, B. Ziaja, and M. Nishikino, Commun. Phys. 2, 150 (2019).

${ }^{8}$ J. Chalupský, L. Juha, J. Kuba, J. Cihelka, V. Hájková, S. Koptyaev, J. Krása, A. Velyhan, M. Bergh, C. Caleman, J. Hajdu, R. M. Bionta, H. Chapman, S. P. Hau-Riege, R. A. London, M. Jurek, J. Krzywinski, R. Nietubyc, J. B. Pelka, R. Sobierajski, J. Meyer-ter-Vehn, A. Tronnier, K. Sokolowski-Tinten, N. Stojanovic, K. Tiedtke, S. Toleikis, T. Tschentscher, H. Wabnitz, and U. Zastrau, Opt. Express 15, 6036 (2007).

${ }^{9}$ J. Chalupský, L. Juha, V. Hájková, J. Cihelka, L. Vyšín, J. Gautier, J. Hajdu, S. P. Hau-Riege, M. Jurek, J. Krzywinski, R. A. London, E. Papalazarou, J. B. Pelka, G. Rey, S. Sebban, R. Sobierajski, N. Stojanovic, K. Tiedtke, S. Toleikis, 
T. Tschentscher, C. Valentin, H. Wabnitz, and P. Zeitoun, Opt. Express 17, 208 (2009).

${ }^{10}$ N. Medvedev, V. Tkachenko, V. Lipp, Z. Li, and B. Ziaja, 4open 1, 3 (2018).

${ }^{11}$ N. Medvedev, H. O. Jeschke, and B. Ziaja, New J. Phys. 15, 015016 (2013).

${ }^{12}$ F. Tavella, H. Höppner, V. Tkachenko, N. Medvedev, F. Capotondi, T. Golz, Y. Kai, M. Manfredda, E. Pedersoli, M. J. Prandolini, N. Stojanovic, T. Tanikawa, U. Teubner, S. Toleikis, and B. Ziaja, High Energy Density Phys. 24, 22 (2017).

${ }^{13}$ R. Follath, T. Koyama, V. Lipp, N. Medvedev, K. Tono, H. Ohashi, L. Patthey, M. Yabashi, and B. Ziaja, Sci. Rep. 9, 2029 (2019).

${ }^{14}$ J. Chalupský, J. Krzywinski, L. Juha, V. Hájková, J. Cihelka, T. Burian, L. Vyšín, J. Gaudin, A. Gleeson, M. Jurek, A. R. Khorsand, D. Klinger, H. Wabnitz, R. Sobierajski, M. Störmer, K. Tiedtke, and S. Toleikis, Opt. Express 18, 27836 (2010).

${ }^{15}$ J. Chalupský, T. Burian, V. Hájková, L. Juha, T. Polcar, J. Gaudin, M. Nagasono, R. Sobierajski, M. Yabashi, and J. Krzywinski, Opt. Express 21, 26363 (2013).

${ }^{16} \mathrm{~N}$. Medvedev and B. Ziaja, Sci. Rep. 8, 5284 (2018).

${ }^{17}$ J. C. Meyer, A. K. Geim, M. I. Katsnelson, K. S. Novoselov, T. J. Booth, and S. Roth, Nature 446, 60 (2007).

${ }^{18}$ A. K. Geim and K. S. Novoselov, Nat. Mater. 6, 183 (2007).

${ }^{19} \mathrm{~J}$. Hopster, R. Kozubek, B. Ban-d'Etat, S. Guillous, H. Lebius, and M. Schleberger, 2D Mater. 1, 011011 (2014).

${ }^{20}$ R. Zan, Q. M. Ramasse, R. Jalil, T. Georgiou, U. Bangert, and K. S. Novoselov, ACS Nano 7, 10167 (2013).

${ }^{21}$ S. Y. Zhou, Ç. Ö. Girit, A. Scholl, C. J. Jozwiak, D. A. Siegel, P. Yu, J. T. Robinson, F. Wang, A. Zettl, and A. Lanzara, Phys. Rev. B 80, 121409 (2009).

${ }^{22}$ E. Akcöltekin, T. Peters, R. Meyer, A. Duvenbeck, M. Klusmann, I. Monnet, H. Lebius, and M. Schleberger, Nat. Nanotechnol. 2, 290 (2007).

${ }^{23}$ V. Vozda, N. Medvedev, J. Chalupský, J. Čechal, T. Burian, V. Hájková, L. Juha, M. Krůs, and J. Kunc, Carbon 161, 36 (2020).

${ }^{24}$ A. Gao, C. J. Lee, and F. Bijkerk, J. Appl. Phys. 116, 054312 (2014).

${ }^{25}$ A. Roberts, D. Cormode, C. Reynolds, T. Newhouse-Illige, B. J. Leroy, and A. S. Sandhu, Appl. Phys. Lett. 99, 051912 (2011).

${ }^{26}$ E. Gruber, R. A. Wilhelm, R. Pétuya, V. Smejkal, R. Kozubek, A. Hierzenberger, B. C. Bayer, I. Aldazabal, A. K. Kazansky, F. Libisch, A. V. Krasheninnikov, M. Schleberger, S. Facsko, A. G. Borisov, A. Arnau, and F. Aumayr, Nat. Commun. 7, 13948 (2016).

${ }^{27} \mathrm{P}$. Yeh, Optical Waves in Layered Media, Wiley Series in Pure and Applied Optics (Wiley, New York, 2005).

${ }^{28}$ B. Ziaja, R. A. London, and J. Hajdu, J. Appl. Phys. 97, 064905 (2005).

${ }^{29}$ M. Harmand, R. Coffee, M. R. Bionta, M. Chollet, D. French, D. Zhu, D. M. Fritz, H. T. Lemke, N. Medvedev, B. Ziaja, S. Toleikis, and M. Cammarata, Nat. Photonics 7, 215 (2013).

${ }^{30}$ R. Riedel, A. Al-Shemmary, M. Gensch, T. Golz, M. Harmand, N. Medvedev, M. J. Prandolini, K. Sokolowski-Tinten, S. Toleikis, U. Wegner, B. Ziaja, N. Stojanovic, and F. Tavella, Nat. Commun. 4, 1731 (2013).

${ }^{31}$ D. E. Cullen, in A Survey of Atomic Binding Energies for Use in EPICS2017, Vienna, 2018.
${ }^{32}$ Y.-K. Kim and M. E. Rudd, Phys. Rev. A 50, 3954 (1994).

${ }^{33}$ N. Medvedev, V. Tkachenko, and B. Ziaja, Contrib. Plasma Phys. 55, 12 (2015).

${ }^{34} \mathrm{M}$. Toufarová, V. Hájková, J. Chalupský, T. Burian, J. Vacík, V. Vorlíček, L. Vyšín, J. Gaudin, N. Medvedev, B. Ziaja, M. Nagasono, M. Yabashi, R. Sobierajski, J. Krzywinski, H. Sinn, M. Störmer, K. Koláček, K. Tiedtke, S. Toleikis, and L. Juha, Phys. Rev. B 96, 214101 (2017).

${ }^{35}$ N. Medvedev, Z. Li, V. Tkachenko, and B. Ziaja, Phys. Rev. B 95, 014309 (2017).

${ }^{36}$ V. Tkachenko, N. Medvedev, Z. Li, P. Piekarz, and B. Ziaja, Phys. Rev. B 93, 144101 (2016).

${ }^{37}$ C. H. Xu, C. Z. Wang, C. T. Chan, and K. M. Ho, J. Phys.: Condens. Matter 4, 6047 (1992).

${ }^{38}$ J. Gaudin, N. Medvedev, J. Chalupský, T. Burian, S. Dastjani-Farahani, V. Hájková, M. Harmand, H. O. Jeschke, L. Juha, M. Jurek, D. Klinger, J. Krzywinski, R. A. Loch, S. Moeller, M. Nagasono, C. Ozkan, K. Saksl, H. Sinn, R. Sobierajski, P. Sovák, S. Toleikis, K. Tiedtke, M. Toufarová, T. Tschentscher, V. Vorlíček, L. Vyšín, H. Wabnitz, and B. Ziaja, Phys. Rev. B 88, 060101(R) (2013).

${ }^{39}$ H. O. Jeschke, M. E. Garcia, and K. H. Bennemann, Appl. Phys. A 69, S49 (1999). ${ }^{40}$ B. F. Murphy, T. Osipov, Z. Jurek, L. Fang, S.-K. Son, M. Mucke, J. H. D. Eland, V. Zhaunerchyk, R. Feifel, L. Avaldi, P. Bolognesi, C. Bostedt, J. D. Bozek, J. Grilj, M. Guehr, L. J. Frasinski, J. Glownia, D. T. Ha, K. Hoffmann, E. Kukk, B. K. McFarland, C. Miron, E. Sistrunk, R. J. Squibb, K. Ueda, R. Santra, and N. Berrah, Nat. Commun. 5, 4281 (2014).

${ }^{41}$ A. Laitinen, M. Oksanen, A. Fay, D. Cox, M. Tomi, P. Virtanen, and P. J. Hakonen, Nano Lett. 14, 3009 (2014).

${ }^{42}$ E. Pomarico, M. Mitrano, H. Bromberger, M. A. Sentef, A. Al-Temimy, C. Coletti, A. Stöhr, S. Link, U. Starke, C. Cacho, R. Chapman, E. Springate, A. Cavalleri, and I. Gierz, Phys. Rev. B 95, 024304 (2017).

${ }^{43}$ S. Krylow, F. V. Hernandez, B. Bauerhenne, and M. E. Garcia, Phys. Rev. B 101, 205428 (2020).

${ }^{44}$ N. Medvedev and I. Milov, Phys. Rev. B 102, 064302 (2020).

${ }^{45}$ Z. Lin, L. Zhigilei, and V. Celli, Phys. Rev. B 77, 075133 (2008).

${ }^{46}$ C. H. Lui, K. F. Mak, J. Shan, and T. F. Heinz, Phys. Rev. Lett. 105, 127404 (2010).

${ }^{47}$ Y. D. Fomin and V. V. Brazhkin, Carbon 157, 767 (2020).

${ }^{48}$ B. L. Henke, E. M. Gullikson, and J. C. Davis, At. Data Nucl. Data Tables 54, 181 (1993).

${ }^{49}$ B. Andonovic, A. Grozdanov, P. Paunović, and A. T. Dimitrov, Micro Nano Lett. 10, 683 (2015).

${ }^{50}$ S. M. Clark, K.-J. Jeon, J.-Y. Chen, and C.-S. Yoo, Solid State Commun. 154, 15 (2013).

${ }^{51}$ M. S. Seehra, V. Narang, U. K. Geddam, and A. B. Stefaniak, Carbon 111, 380 (2017).

${ }^{52}$ M. Gulde, S. Schweda, G. Storeck, M. Maiti, H. K. Yu, A. M. Wodtke, S. Schafer, and C. Ropers, Science 345, 200 (2014).

${ }^{53}$ M. Bruna and S. Borini, Appl. Phys. Lett. 94, 031901 (2009).

${ }^{54}$ H. S. Skulason, P. E. Gaskell, and T. Szkopek, Nanotechnology 21, 295709 (2010). 